\title{
Application of a Three-Tiered Instruction Model for Japanese Second-Grade Students to Improve Accuracy and Fluency of Multiplication Facts
}

\author{
Wataru NodA $^{1, *}$ and Junko TANAKA-MATSUMI ${ }^{2}$ \\ ${ }^{1}$ Research Center for Child Mental Development, Hamamatsu University School of Medicine, Japan \\ ${ }^{2}$ Department of Integrated Psychological Science, Kwansei Gakuin University, Japan
}

\begin{abstract}
We examined the impact of three-tiered instruction on multiplication facts for Japanese second-grade public elementary students $(N=59)$. In Tier 1 (about three months), all students received regular classroom instruction from their classroom teachers. We monitored the accuracy and fluency of their performance on multiplication facts. In Tier 2 (about one month), teachers conducted a 10-minute supplemental instruction (flash card practice) $(n=17)$. In Tier 3 (about three weeks), students $(n=5)$ practiced multiplication facts through the "covercopy-compare" method and a timed trial procedure. We calculated the percentage of correctly answered problems and assessed the number of correct and incorrect problems per minute in 1-minute timed trials. A multiple probe design across instructional materials was used to evaluate the effect of Tier 3 instruction. Fifty-three of the 59 students demonstrated mastery of all multiplication facts during Tier 1 and Tier 2. However, the remaining six students including one who did not complete the study did not master all multiplication facts in Tier 3. Results of this study suggest concrete practical issues that should be addressed in future practical research.
\end{abstract}

Key Words: three-tiered instruction, cover-copy-compare, fluency, accuracy, multiplication facts

\section{Introduction}

In the current educational system, $4.5 \%$ of students in regular elementary and junior high school classrooms have been reported to exhibit academic difficulty, based on a national survey of schoolchildren (Ministry of Education, Culture, Sports, Science \& Technology, 2003). In recent years, there has been increasing demand for effective educational practices to assure acquisition of basic academic skills in Japanese regular classrooms. Some researchers showed that tiered instruction model from the response to intervention/instruction (RtI) were effective educational practices that can be used in Japanese education (e.g., Kaizu, Tanuma, \& Hiraki, 2009; Kaizu, Tanuma, Hiraki, Ito, \& Vaughn, 2008). This study applied a three-tiered instruction model to improve multiplication facts performance in Japanese

\footnotetext{
* Corresponding Author

Mailing Address: 1-20-1 Handayama, Higashi-ku, HamamatsuCity 431-3192, Japan

E-mail Address: watarunoda@gmail.com

Received April 13, 2013, Accepted October 26, 2013
}

second-grade classrooms.

The response to intervention/instruction (RtI) model is an approach that aims to enhance learning for all students, including those who are at risk but not identified as having disability (Burns \& VanDerHeyden, 2006). RtI is based on applied behavior analysis, behavioral assessment, and behavioral consultation (Reschly \& Bergstrom, 2009). The RtI model typically includes three progressively intensive tiers (VanDerHeyden \& Burns, 2010). The first tier delivers quality instruction for all students in regular classrooms and monitors and screens their progress. The second tier consists of supplemental instruction, usually in small groups, and includes frequent progress monitoring for problem analysis. The third tier is designed to provide intensive instruction that is usually conducted individually and with more frequent progress monitoring to identify an intervention method that will accelerate the learning of individual students. Previous researches demonstrated the effectiveness of the tiered instruction to improve mathematics skills (e.g., Ardoin, Witt, Connell, \& Koenig, 2005; VanDerHeyden, 2010). For example, Ardoin et al. (2005) applied a three-tiered RtI model 
to elementary school mathematics (subtraction) instruction. During Tier 1, they conducted class-wide screening in mathematics using curriculum-based measurement probes and identified students in need of math intervention. The screening data showed that all 14 students in the class performed at low levels of fluency on subtraction problems with regrouping and did not reach the suggested mastery rates of fluency (4th-grade instructional level, 40-88 digits correct in 2 minutes) suggested by Shapiro (1996). Therefore, a class-wide intervention for subtraction was conducted for all 14 students during Tier 2 prior to the Tier 3 individualized intervention. The class-wide behavioral instruction procedure included modified instruction from a Direct Instruction manual (Stein, Silbert, \& Carnine, 1997), the cover-copy-compare method (CCC; Skinner, Turco, Beatty, \& Rasavage, 1989), a 2-minute timed trial, and another 2-minute timed trial with beat-your-score contingency. CCC is a self-managed instruction method that includes reading the correct answer, covering the answer to respond, and self-marking. Four students whose performance remained low following Tier 2 participated in the Tier 3 instruction. During Tier 3, Ardoin et al. (2005) applied peer tutoring alone or peer tutoring and CCC to improve subtraction performance. With the exception of one student, Tier 3 instruction increased the students' fluency in subtraction. Previous studies using RtI model extended previous findings that supported the use of behaviorally based instruction to improve the academic performance of students with academic difficulties.

When we introduce a new system or model to schools, we need to carefully consider its acceptability by teachers. Erchul and Martens (2002) suggested two key factors leading to success of intervention in schools: (a) whether all teachers sufficiently understand the method and process of the intervention; (b) whether all teachers think of the method and process of the intervention as completely acceptable. Therefore, we need to use brief, easy to understand assessment tools and instructional materials that the teachers are experienced with when we introduce a new instructional model to schools.

This practical research aimed at introducing a three-tiered instruction model to Japanese secondgrade classrooms to improve multiplication performance and to evaluate the impact of the model. We adapted the existing assessment method in Tier 1 and
2 rather than introducing an entirely new tool, and we created a manual for all of the instructional procedures in Tier 3. Throughout the study, we used a timed trial procedure for the assessment, and we used the CCC (Skinner et al., 1989) as an instructional procedure during Tier 3 . We worked collaboratively with classroom teachers to implement a tiered instruction and to collect assessment data.

\section{Method}

\section{Participants and Setting}

Fifty-nine second-grade students in two Japanese public elementary school classrooms participated in this study. A total of 352 students attended the school. The school was located in the suburbs of a city in Japan with a population of 154,000 . According to the principal of the school, majority of the students came from low-income families. This school conducted a standardized achievement test for mathematics (Kyouken-shiki Standardized Achievement Test; Tatsuno, Ishida, Hattori, Moriyama, Tanaka, Nakata, Natsusaka, Hosomizu, \& Yamamoto, 2009) at the end of the school year (during the latter half of this study). This achievement test uses a standard score to assess students' academic achievement, with a mean standard score of 50 and a standard deviation of 10 . The mean standard score of the secondgrade students in this school was 49.9. All secondgrade students participated in the Tier 1 instruction, and 17 students who did not master multiplication facts participated in the Tier 2 instruction. Of the six students who did not master multiplication facts in Tier 2, five students participated in Tier 3 afterschool instruction with parental consent. One of the six students did not participate in the Tier 3 instruction due to lack of parental consent. Although this student no longer participated in our study at this point, she received help she could find from the classroom teacher (e.g., individual extra instruction in recess time). The standard scores on the achievement test for mathematics of these five students were as follows: 32 for student A, 15 for student B, 15 for student $\mathrm{C}, 44$ for student $\mathrm{D}$, and 15 for student $\mathrm{E}$.

Figure 1 represents the framework of the threetiered instruction model. We conducted this study from September to December 2009 for Tier 1, from January to February 2010 for Tier 2, and in March 2010 for Tier 3. Two homeroom teachers (Teachers A 


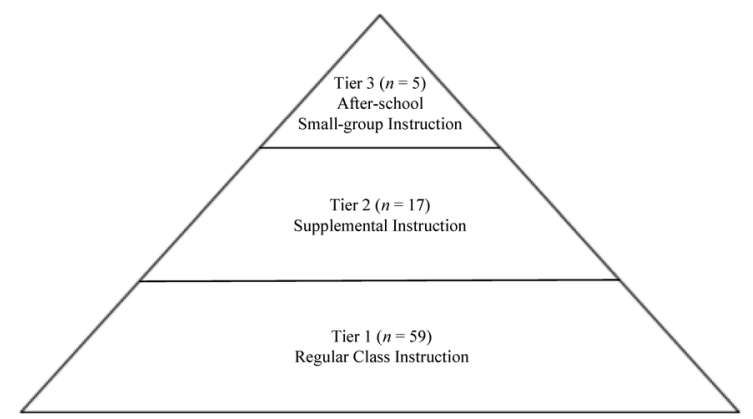

Fig. 1 Framework of the Three-tiered Instruction Model in This Study

and B) and an additional homeroom teacher (Teacher C) conducted Tier 1 instruction in each classroom in the form of regular group instruction for 45 minutes per session. Teacher $\mathrm{C}$ mainly taught mathematics classes. None of the teachers were trained as special education teacher. In Tier 1 , teacher $\mathrm{C}$ taught the students, and each homeroom teacher supported the students individually in a team-teaching format. Tier 2 instruction was conducted approximately 4 times per week in a room adjacent to the classroom during the last 10 minutes of the mathematics class. Each student practiced multiplication facts independently using flash cards (by viewing the multiplication fact problem on the front of the card, saying the answer to the problem, and then checking the answer on the back of the card). Teacher $\mathrm{C}$ implemented Tier 3 instruction 3 times per week for approximately 30 minutes in the same room as Tier 2 in the form of after-school small-group sessions. Teacher $\mathrm{C}$ taught students using the CCC procedure. In the Tier 3 instruction, we collected follow-up data one week after the end of the instruction.

This study was conducted as a collaboration project between the authors and the participating schoolteachers to improve children's basic academic skills. The first author supported second-grade students with behavioral or academic difficulties in the classroom weekly by directing children's attention to their classroom teacher, prompting them to engage in the academic task, and praising their on-task behaviors. The second author served as a special education consultant to the school as well as supervisor for the first author. Informal direct observation of the first author suggested that second-grade classrooms had many students with academic difficulties. Therefore, the authors proposed this study as a collaboration project. The first author explained the purposes, pro- cedures, and publication of this study to the school principal and the participating teachers and they agreed to participate in this study. Following the request of the principal, we obtained the consents from the parents of students who participated in the Tier 3 after-school instruction.

\section{Target Academic Behaviors and Dependent Variables}

The target academic behaviors of this study were reciting multiplication facts and writing the answers to multiplication problems in 1-minute timed trials. There were four mastery criteria for multiplication facts. The first three criteria concerned accuracy: reciting 10 multiplication facts in order with no error for each number below 10 (e.g., $3 \times 1=$ $3,3 \times 2=6, \ldots 3 \times 10=30$ ); reciting 10 multiplication facts in reverse order with no error for each number (e.g., $3 \times 10=30,3 \times 9=27, \ldots 3 \times 1=3$ ); and reciting 10 multiplication facts in random order with no error for each number (e.g., $3 \times 3=9,3 \times 6=18, \ldots 3 \times 8=24$ ). The last criterion was the fluency criterion: writing 20 answers to multiplication problems with no error in 1-minute timed trials. We used all multiplication fact sets (multiples of 1 to 9) in Tier 1 and Tier 2. We used the number of students who met all four mastery criteria as a dependent variable in Tiers 1 and 2. In Tier 3 , we calculated the percentage of correct problems in 1-minute timed trials as an accuracy measure. In addition, we measured the number of correct and incorrect problems per minute in 1-minute timed trials as a fluency measure. We selected the multiples of 2,3, and 4 of multiplication facts as instructional materials based on the participants' mastery level during Tier 3.

\section{Procedures}

Tier 1 instruction. Teacher $\mathrm{C}$ and each homeroom teacher (Teachers $\mathrm{A}$ and $\mathrm{B}$ ) conducted regular class instruction (teaching the concepts of multiplication, problem solving for multiplication, and practicing multiplication facts using flash cards) in the form of team teaching. In the latter half of each class, the teachers assessed the accuracy and fluency of students' multiplication facts for approximately 20 minutes. In the accuracy assessment, the students orally recited multiplication facts (in order, in reverse order, or in random order), and the teachers checked their accuracy. In the fluency assessment, the students solved multiplication problems in writing as 
fast as possible during 1-minute timed trials.

Tier 2 instruction. Seventeen of the 59 students who did not master multiplication facts during Tier 1 participated in the Tier 2 instruction. In Tier 2, the students practiced multiplication facts independently using flash cards. Teacher $\mathrm{C}$ conducted the accuracy and fluency assessments. Tier 2 instruction took approximately 10 minutes.

Tier 3 instruction. Five students who did not master multiplication facts despite their enrollment in Tier 2 instruction participated in the Tier 3 afterschool instruction. Informal observation by the first author indicated that five students engaged most of their time in off-task behavior during Tier 2 instruction. This might be because students were not told explicitly how many times they should repeat the flash card practice, and independent practice with flash cards did not provide adequate immediate feedback to students' responses. Therefore, we used a CCC procedure in a choral response format to increase their on-task behavior. In Tier 3, instruction proceeded in the following order: a 1-minute timed trial, CCC, and a 1-minute timed trial. At the beginning and end of the instruction, the students solved multiplication facts in writing as fast as possible in 1-minute timed trials, and the teacher provided feedback (the number of correct and incorrect problems). CCC is a self-managed instruction method that includes reading the problems accompanied with their answers, solving the problems, and immediate self-marking. We modified the typical CCC procedures (e.g., Skinner et al., 1989) to utilize the "ku-ku" learning method for multiplication facts used in Japanese education. In the "ku-ku" method, students practice by reciting the multiplication facts in a way that is phonologically easy to memorize. For example, they pronounce " 2 times 2 equals 4 " as "nini-n-ga-shi". The CCC procedure in this study was composed of 6 steps: (1) reading the problem and the answer for the multiplication facts three times, (2) folding the left side of the practice sheet and covering the problem and answer, (3) reciting the multiplication facts three times; (4) writing the multiplication facts on the right side of the sheet, (5) uncovering the problem and answer, and (6) evaluating their own responses. Noda (2011) demonstrated the effect of this modified version of CCC on multiplication facts for second-grade elementary students. In the CCC instruction in this study, the students practiced mul- tiplication facts in a choral response format in the classroom to increase their on-task behavior. The teacher orally cued the timing of each of the six CCC steps. In the last part of each session, the teacher gave all of the students stickers to reward their participation in the Tier 3 after-school sessions. We created a manual of all of the instructional procedures, and teacher $\mathrm{C}$ used this manual to follow the instructional protocol during the Tier 3 instruction.

\section{Experimental Design}

In Tiers 1 and 2, we evaluated the effects of instruction using the number of students who met all mastery criteria as a dependent variable. We used a multiple probe design across instructional materials (Barlow \& Hersen, 1984) to evaluate the effects of Tier 3 instruction. In the probe, we assessed students' fluency in multiplication facts in 1-minute timed trials without feedback. We collected follow-up data on 3 of the 5 students one week after the end of the instruction.

\section{Interscorer Agreement and Procedural Integrity}

The first author and a graduate student who was not informed of the purpose of the study scored the students' performance in the Tier 3 instruction. Two scorers checked all five students' worksheets (Student A: 21 worksheets, Student B: 20 worksheets, Student C: 23 worksheets, Student D: 18 worksheets, Student E: 25 worksheets). The interscorer agreement (\%) of the correct and incorrect problems for all students was calculated by dividing the number of agreements by the number of agreements and disagreements and multiplying by 100 . The interscorer agreement for the correct and incorrect problems for each student was $100 \%$.

We created a manual of procedures for Tier 3, and Teacher $\mathrm{C}$ used the manual to follow the instructional protocol during the Tier 3 instruction. Approximately once per week, the first author informally observed whether Teacher $\mathrm{C}$ followed the instructional protocol precisely and followed the manual.

\section{Teacher C's Acceptability Ratings of CCC (Tier 3 Instruction)}

We used the Intervention Rating Profile-15 (Martens, Witt, Elliott, \& Darveaux, 1985) to assess Teacher C's acceptability of CCC instruction in Tier 3. 
Teacher C, who implemented the Tier 3 instruction, responded to the questionnaire on a 6-point scale ( $1=$ strongly disagree, $6=$ strongly agree).

\section{Results}

\section{Tier 1 and Tier 2 Instruction}

Figure 2 shows the number of students who met all mastery criteria for each multiplication facts series in the Tier 1 and Tier 2 instruction. In Tier 1, approximately 40 of the 59 students ( 37 in multiples of 5, 40 in 2, 42 in 3, 36 in 4, 45 in 6, 27 in 7, 24 in 8, and 50 in 9) met all mastery criteria for each multiplication facts series during the early second or third sessions. The number of students who met the mastery criteria gradually increased. However, 17 of the 59 students (28.9\%) ultimately failed to meet all of the mastery criteria during the class-wide instruction in Tier 1.

During the Tier 2 instruction, 17 students independently practiced multiplication facts with flash cards. Eleven of the 17 students (64.7\%) achieved all of the mastery criteria for all multiplication facts series, and as a result, number of students who met all mastery criteria increased from 42 to 53 . However,

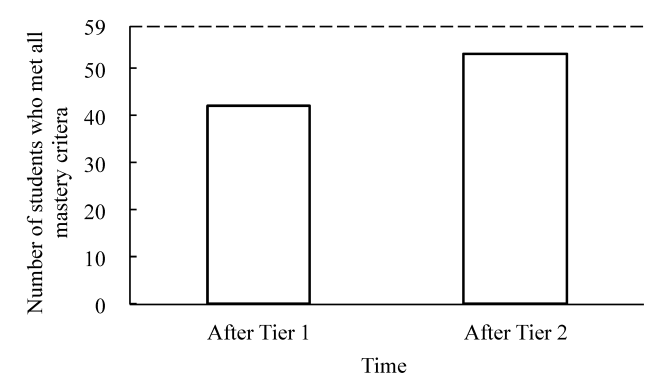

Fig. 2 Number of Students Who Met All Mastery Criteria in Tier 1 and Tier 2 Instruction

Note. A broken line represents the total number of students $(N=59)$. the remaining 6 students failed to master multiplication facts. The informal direct observation by the first author indicated that these six students exhibited off-task behavior, such as talking with other students, walking around the room, and playing with their pencils or erasers for at least half of the Tier 2 instruction. Five of the six students participated in the Tier 3 instruction with parental consent. The other students who met all of the mastery criteria during the Tier 1 instruction engaged in other mathematics instruction with their homeroom teachers.

\section{Tier 3 Instruction}

We evaluated the effectiveness of Tier 3 instruction using a multiple probe design across three instructional materials (multiples of 2, 3, and 4 in multiplication sheets). Table 1 presents the descriptive statistics of the percentage of correct problems (accuracy measure) during the Tier 3 instruction of five students. Figures 3 through 7 show the number of correct and incorrect problems per minute in the 1 -minute timed trials (fluency measure) during the Tier 3 instruction of five students. In each figure, the upper panel shows the result of the multiples of 2 , the middle panel shows the result of the multiples of 3 , and the bottom panel shows the result of the multiples of 4 . All five students were on-task during Tier 3 instruction (CCC and timed trials).

Students A, D, and E improved their accuracy and fluency for multiplication facts in all instructional materials. Student B improved her accuracy and fluency for multiplication facts in the multiple of 2, but her number of incorrect problems per minute did not decrease in the multiples of 3 and 4 . Informal direct observation during the instruction and an analysis of the student's response pattern by the first author indicated that the student solved problems quickly but

Table 1 Mean and Standard Deviations of Percentage of Correct Problems of 1-minute Timed Trials in Each Phase During Tier 3 Instruction for 5 Students

\begin{tabular}{|c|c|c|c|c|c|c|c|}
\hline & \multicolumn{3}{|c|}{ Multiples of 2} & \multicolumn{2}{|c|}{ Multiples of 3} & \multicolumn{2}{|c|}{ Multiples of 4} \\
\hline & Baseline & Instruction & Follow-up & Baseline & Instruction & Baseline & Instruction \\
\hline & Mean $(S D)$ & Mean $(S D)$ & Mean $(S D)$ & Mean (SD) & Mean $(S D)$ & Mean (SD) & Mean $(S D)$ \\
\hline Student A & $100(-)$ & $100(0.97)$ & $100(-)$ & $80(17.69)$ & $81(20.4)$ & $65(5.10)$ & $77(16.4)$ \\
\hline Student B & $93(-)$ & $90(2.04)$ & $98(-)$ & $65(8.54)$ & $68(4.75)$ & $41(.84)$ & $42(-)$ \\
\hline Student C & $9(-)$ & $99(2.63)$ & 100 & $65(49)$ & $66(22.9)$ & $27(1.2)$ & $38(8.36)$ \\
\hline Student D & $100(-)$ & $100(0)$ & - & $99(2.41)$ & $100(0)$ & $93(-)$ & $97(1.82)$ \\
\hline Student E & $100(-)$ & $93(5.7)$ & - & $69(13)$ & $75(16)$ & $30(-)$ & $53(6.9)$ \\
\hline
\end{tabular}




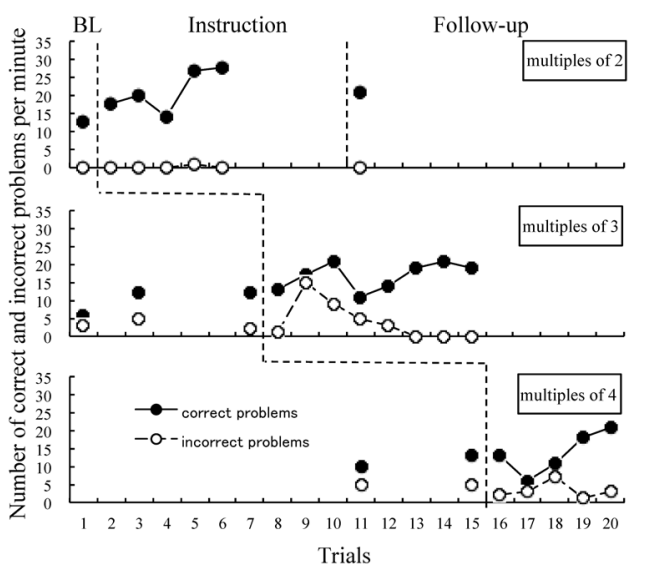

Fig. 3 Student A's Number of Correct and Incorrect Problems per Minute

Note. $\mathrm{BL}=$ Baseline.

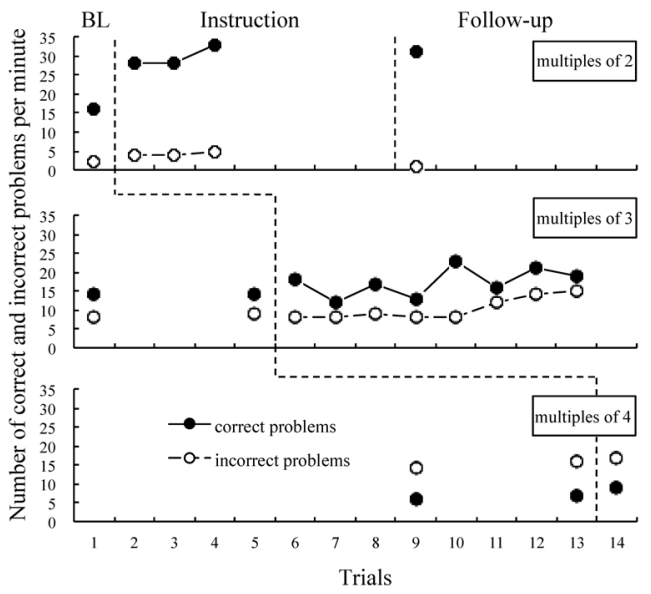

Fig. 4 Student B's Number of Correct and Incorrect Problems per Minute

Note. $\mathrm{BL}=$ Baseline.

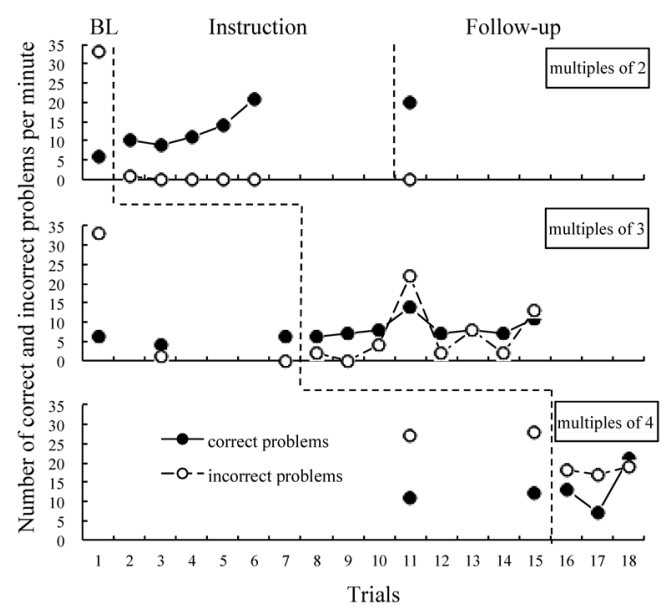

Fig. 5 Student C's Number of Correct and Incorrect Problems per Minute

Note. $\mathrm{BL}=$ Baseline.

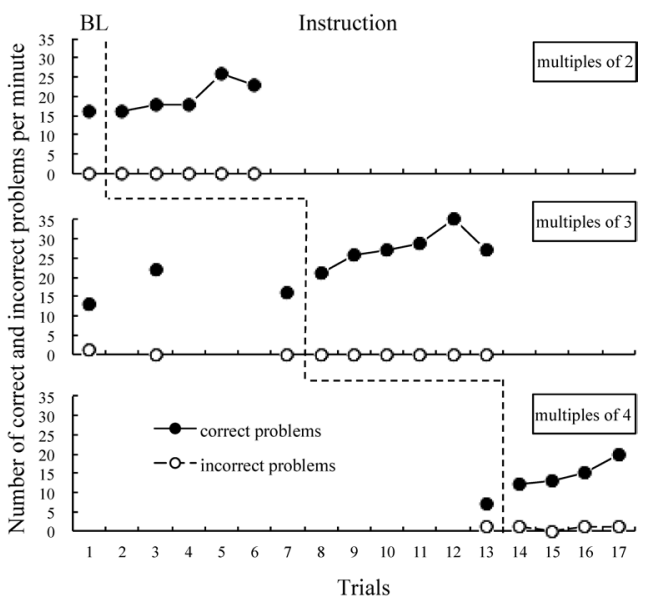

Fig. 6 Student D's Number of Correct and Incorrect Problems per Minute

Note. $\quad \mathrm{BL}=$ Baseline.

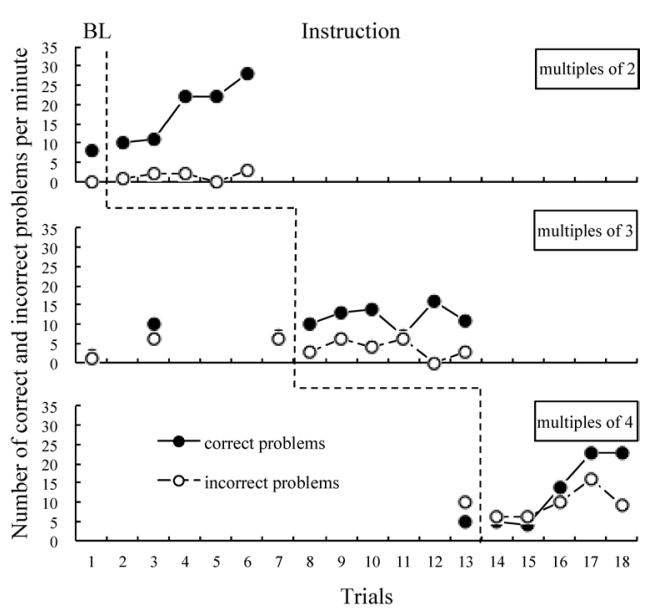

Fig. 7 Student E's Number of Correct and Incorrect Problems per Minute

Note. $\quad \mathrm{BL}=$ Baseline.

not accurately. Student C improved his accuracy and fluency in the multiples of 2 , but the effect of the instruction was unclear in the multiples of 3 and 4 . An analysis of the student C's response pattern indicated that the number of skipping response increased from the start of the instruction for multiples of 3 . The student answered four or five particular problems the student learned and skipped problems that were still not memorized in the multiples of 3 and 4 .

\section{Teacher C's Acceptability Ratings of CCC in Tier 3}

Teacher C, who implemented the Tier 3 instruction, rated the acceptability of CCC (Intervention Rating Profile-15) on a 6-point scale, with 6 indicating "strongly agree" and 1 indicating "strongly disagree". The teacher rated 14 of the 15 items positive- 
ly with a score of 5 or higher but rated the item on appropriateness for a variety of children with a score of 4 .

\section{Discussion}

The results of the present study demonstrates the feasibility of adapting the three-tiered instruction model in regular classrooms at a Japanese public elementary school through the collaboration of outside professionals and school teachers. We highlight the main findings of the three-tiered instructional method that was designed to help all students improve their academic performance.

When a new instructional model is introduced to schools, it is important to use brief and easy to understand assessment tools and instructional materials. In addition, group-based instruction is emphasized due to Japan's collectivistic cultural values. Therefore, teachers need an instructional system that includes effective instruction for all students in regular classrooms and graded support procedures for struggling students. The present study demonstrated the applicability of a three-tiered instruction model in Japanese regular classrooms. We developed user-friendly assessment and instructional procedures and collaborated with teachers to implement the tiered instruction. Our study mainly focused on fluency of computation skills, which has not been systematically examined in Japanese school settings. During the three-tiered instruction program, students' accuracy and fluency in multiplication facts improved. The teacher also responded positively regarding the acceptability of the CCC instruction in Tier 3 instruction by providing excellent ratings. Introducing the three-tiered instruction model led to data-based screening for the students who required additional support and graded support practices corresponding to the individual's educational needs.

The results of the present study demonstrated that the three-tiered instruction model is applicable in Japanese classrooms. However, further research is required on three important points: assessment, intervention, and pragmatic issues. First, the three-tiered instruction model requires measurement tools for continual assessment and empirically based instruction. The RtI model (e.g., Burns \& VanDerHeyden, 2006) and the Multilayer Instruction Model (Kaizu et al., 2008) include measurement tools for screen- ing and evaluating the effect of the instruction. The present study adopted a fluency measure assessed by 1-minute timed trials. The fluency measure of computation skills is sensitive to individual differences and predicts the standardized achievement test score for mathematics among Japanese regular elementary students (Noda, 2011). Further research should examine the benchmarks of Japanese students' academic skills using this fluency measure, which would lead to data-based decision making in Japanese educational practices.

Second, we must develop more effective instructional procedures to remedy academic problems. Five students who participated in Tier 3 instruction were able to increase their accuracy and fluency of multiplication facts during Tier 3 instruction compared to that during Tier 1 and Tier 2 instruction. However, they did not master all multiplication facts through this three-tiered instruction model. According to the result of a standardized achievement test for mathematics, they had not adequately acquired academic skills in mathematics. To maximize their learning rate, we need to assess what academic skills they have, and which intervention is most effective for a particular student. Witt and VanDerHeyden (2007) utilized the functional academic assessment (Duhon, Noell, Witt, Freeland, Dufrene, \& Gilbertson, 2004) and Instructional Hierarchy (Haring, Lovitt, Eaton, \& Hansen, 1978) as useful assessment frameworks for intervention selection within their RtI model. We need to investigate the utility of these assessment frameworks in Japanese school settings as well as attempt to develop our own assessment tools.

Finally, future research should address pragmatic issues regarding the transition to this three-tiered model. In this study, students who did not master multiplication facts via Tier 1 proceeded to Tier 2 instruction. Students who mastered the skills in Tier 1 moved on to the next curriculum while Tier 2 instruction was conducted. This transition method may exacerbate the divide between high-achieving students and students who require more intensive instructional time via Tier 2 and Tier 3 methods. Developing a homogeneous group and differentiating instruction according to students' mastery levels (e.g., Johnson \& Street, 2012) is a promising approach to resolve this problem. Precise assessment and measurement system for academic skills is necessary in order to give effective instruction to students and to 
achieve a homogenious level. The development of a progress monitoring system for each student in regular classrooms is an important first step to implementing multi-tiered instruction.

In conclusion, the present study demonstrated the feasibility of a tiered instruction model that can be used in Japanese classrooms to benefit all students at different levels of academic performance. In addition, the results of this study suggest the important practical issues that should be addressed in future research. More practical research which use data-based decision making process in schools are required to promote all students' academic learning.

\section{Acknowledgments}

We thank the teachers and students who participated in this study. We presented a preliminary report of this study at the 36th Annual Convention of the Association for Behavior Analysis International, San Antonio, U.S. (June 2010).

This research was supported by a Grant-in-Aid from the Japan Society for the Promotion of Science to the first author.

\section{References}

Ardoin, S. P., Witt, J. C., Connell, J. E., \& Koenig, J. L. (2005) Application of a three-tiered response to intervention model for instructional planning, decision making, and the identification of children in need of services. Journal of Psychoeducational Assessment, 23, 362-380.

Barlow, D. H. \& Hersen, M. (Eds.) (1984) Single-case experimental designs: Strategies for studying behavior change (2nd ed.). Pergamon Press, Oxford.

Burns, M. K. \& VanDerHeyden, A. M. (2006) Using response to intervention to assess learning disabilities: Introduction to the special series. Assessment for Effective Intervention, 32, 3-5.

Duhon, G. J., Noell, G. H., Witt, J. C., Freeland, J. T., Dufrene, B. A., \& Gilbertson, D. N. (2004) Identifying academic skills and performance deficits: The experimental analysis of brief assessments of academic skills. School Psychology Review, 33, 429-443.

Erchul, W. P. \& Martens, B. K. (2002) School consultation: Conceptual and empirical bases of practice. New York: Kluwer Academic/Plenum Publishers.

Haring, N. G., Lovitt, T. C., Eaton, M. D., \& Hansen, C. L. (1978) The fourth R: Research in the classroom. Columbus, OH: Merrill.
Johnson, K. R. \& Street, E. M. (2012) From the laboratory to the field and back again: Morningside Academy's 32 years of improving students' academic performance. Behavior Analyst Today, 13, 20-40.

Kaizu, A., Tanuma, M., Hiraki, K., Ito, Y., \& Vaughn, S. (2008) Multilayer instruction model for first-grade students in general education classes: Reading and writing instruction on special morae. Japanese Journal of Educational Psychology, 56, 534-547. (in Japanese)

Kaizu, A., Tanuma, M., \& Hiraki, K. (2009) Intensive instruction on special morae for first-grade struggling readers: The multilayer instruction model (MIM) in general education classes. Japanese Journal of Special Education, 47, 1-12. (in Japanese)

Martens, B. K., Witt, C. J., Elliott, S. N., \& Darveaux, D. X. (1985) Teacher judgments concerning the acceptability of schoolbased intervention. Professional Psychology, Research and Practice, 16, 191-198.

Ministry of Education, Culture, Sports, Science \& Technology (2003) Kongo no tokubetsu shien kyoiku no arikata ni tsuite (Saisyu hokoku). [Prospect of special needs education in the future (Final Report)]. Tokubetsu shien kyoiku no arikata ni kansuru tyosakenkyu kyoryokusya kaigi. (in Japanese)

Noda, W. (2011) Japanese elementary school students' fluency of academic skills: Behavior analytic approach to academic instruction. Unpublished doctoral dissertation. Kwansei Gakuin University. (in Japanese)

Reschly, D. J. \& Bergstrom, M. K. (2009) Response to intervention. In T. Gutkin \& C. Reynolds (Eds.), The handbook of school psychology (4th ed.). Hoboken, NJ: John Wiley \& Sons, 434-459.

Shapiro, E. S. (1996) Academic skills problems. New York: Guilford Press.

Skinner, C. H., Turco, T. L., Beatty, K. L., \& Rasavage, C. (1989) Cover, copy, compare: An intervention for increasing multiplication performance. School Psychology Review, 18, 212-220.

Stein, M., Silbert, J., \& Carnine, D. W. (1997) Designing effective mathematics instruction: A direct instruction approach (3rd ed). Columbus: Prentice Hall.

Tatsuno, T., Ishida, T., Hattori, T., Moriyama, T., Tanaka, H., Nakata, T., Natsusaka, S., Hosomizu, Y., \& Yamamoto, Y. (2009) Kyo-ken shiki hyojun gakuryoku kensa NRT. [Kyokenshiki norm referenced standardized achievement test]. Tokyo: Toshobunka. (in Japanese)

VanDerHeyden, A. M. (2010) Analysis of universal academic data to plan, implement, and evaluate schoolwide improvement. In G. G. Peacock, R. A. Ervin, E. J. Daly III, \& K. W. Merrell (Eds.), Practical handbook of school psychology: Effective practices for the 21st century. New York: Guilford Press, 33-47. 
VanDerHeyden, A. M. \& Burns, M. K. (2010) Essentials of Response to Intervention. Hoboken, NJ: John Wiley \& Sons, Inc.

Witt, J. C. \& VanDerHeyden, A. M. (2007) The system to enhance educational performance (STEEP): Using science to improve achievement. In S. R. Jimerson, M. K. Burns, \& A. M. VanDerHeyden (Eds.), Handbook of response to intervention: The science and practice of assessment and intervention. New York: Springer, 343-353. 